\title{
Evaluación de programas online de capacitación docente sobre innovación y competencias digitales durante la Covid-19: \#webinarsUNIA
}

\section{(Evaluation of Online Teacher Training Programs on Innovation and Digital Competences During Covid-19: \#webinarsUNIA)}

\author{
María Sánchez González \\ Universidad Internacional de Andalucía y Universidad de Málaga (España) \\ María Lourdes Miró Amarante \\ Universidad Internacional de Andalucía y Universidad de Sevilla (España) \\ Francisco José Ruiz Rey \\ Universidad de Málaga (España) \\ Manuel Cebrián de la Serna \\ Universidad Internacional de Andalucía y Universidad de Málaga (España)
}

DOI: https://doi.org/10.5944/ried.25.1.30763

Cómo referenciar este artículo:

Sánchez González, M., Miró Amarante, M. L., Ruiz Rey, F. J., y Cebrián de la Serna, M. (2022). Evaluación de programas online de capacitación docente sobre innovación y competencias digitales durante la Covid-19: \#webinarsUNIA. RIED. Revista Iberoamericana de Educación a Distancia, 25(1), pp. 121-140. https:// doi.org/10.5944/ried.25.1.30763

\section{Resumen}

La pandemia de la Covid-19 ha provocado una transformación digital en la docencia universitaria aún por valorar y analizar en todas sus dimensiones. Ante ello, las instituciones han desarrollado diferentes estrategias, también en red, para formar al profesorado. El presente artículo expone un programa universitario, los \#webinarsUNIA, enmarcado en los planes anuales de formación al profesorado de la Universidad desde 2013-14 pero en abierto y sin coste para cualquier persona interesada. Nos centramos en los resultados de la edición 2020-21, con una veintena de seminarios como respuesta a las demandas formativas docentes fruto de la sobrevenida y rápida virtualización de los programas durante la pandemia, y que reunió a 10.933 participantes de diversa procedencia geográfica. Para medir su satisfacción se 
diseñaron encuestas con "alfa de Cronbach" 0,789 que consideraron, entre otras dimensiones, organización, docentes, contenidos y formato de las actividades. La evaluación fue opcional y obtuvo una muestra de 1.908 respuestas (34\% de quienes concluyeron la formación). Los resultados fueron en general muy positivos, atendiendo al principio de pertinencia en los objetivos, necesidades recogidas en un sondeo previo al diseño y oportunidad en el formato. Además, abrieron paso a la generación de nuevos recursos y productos de aprendizaje en formato SPOC de gran utilidad para capacitar sobre competencias digitales, innovación educativa y e-learning a un gran número de docentes en el escenario de la Covid-19.

Palabras clave: educación superior; formación de profesores; competencias digitales; webinar; REA.

\begin{abstract}
The Covid-19 pandemic has caused a digital transformation in university teaching that has yet to be assessed and analyzed in all its dimensions. Faced with this, institutions have developed different strategies, also in network, to train teachers. This paper offers a university program, \#webinarsUNIA, framed in annual university teacher training plans from 2013-14 but open and free of charge for anyone interested. We focused on the results of the 2020-21 edition, with twenty seminars in response to teacher training demands due to the sudden and rapid virtualization of their programs during the pandemic and with a total of 10,933 participants from different geographical origins. To measure their satisfaction, surveys were designed with Cronbach's alpha 0.789, which considered, among other dimensions: organization, teachers, contents and format of the activities. The evaluation was optional and obtained a sample of 1908 responses (34\% of those who completed the training). The outcomes were generally rather positive because they met the principle of relevance in the objectives, gathered in a needs survey prior to the design and timeliness in the format. In addition, they paved the way for the generation of new resources and learning products in SPOC format, which are very useful for training a large number of teachers on digital competences, educational innovation and e-learning in the Covid-19 scenario.
\end{abstract}

Keywords: higher education; teacher training; digital competences; webinar; OER.

En los últimos años asistimos al auge de diversas formas de aprendizaje en abierto y en línea, muchas lideradas desde las Universidades, que con el impulso de repositorios digitales y otros espacios en internet con recursos educativos en abierto (REA) o la participación en redes del tipo OpenCourseWare, han venido aportando al movimiento de acceso abierto. La propia enseñanza universitaria se ha visto contagiada de este movimiento, sumando cursos masivos en línea y en abierto en formato MOOC (del inglés, Massive Open Online Courses) a su oferta de estudios. Hoy encontramos diversas fórmulas "evolucionadas" de los MOOC originarios en cuanto a metodología (Raposo-Rivas et al., 2015) o en cuanto a diseño y formatos (más o menos masivos y de distinta duración y carga lectiva). Comparten en cualquier 
caso el protagonismo del audiovisual, esto es, el vídeo es siempre eje secuencial de los contenidos.

Sin embargo, revisando la numerosa literatura especializada en este fenómeno como López-Meneses et al. (2015); Zancanaro y Domingues (2017); o Albelbisi et al. (2018) - cabe plantear si la democratización esperada del conocimiento compartido no se ha visto comprometida por factores no contemplados en su diseño inicial. Entre éstos, la evaluación de los productos y servicios sobre su diseño pedagógico (GallegoArrufat et al., 2015; Yepes-Baldó et al., 2016; Bournissen et al., 2019); o la usabilidad y satisfacción de los usuarios según distintos formatos (Lemos-de-Carvalho-Junior et al., 2019).

\section{Potencial de lo audiovisual e importancia del contenido}

Estudios recientes consideran que su lenguaje audiovisual ha significado un avance didáctico, pero no basta para promover el compromiso del estudiante y reducir el abandono, debilidad crucial de estos modelos (García-Peñalvo et al., 2017, p. 123). La situación viene a identificar de nuevo la antigua visión griega entre la tecné -la tecnología del video- y la episteme -el conocimiento-. Quizás las claves del equilibrio entre ambas dimensiones están en la propuesta de Deng et al. (2020), quienes tras analizar los resultados del aprendizaje (finalización y calidad percibida) sobre muestras y contextos de enseñanza diversos concluyen que para que las actividades de aprendizaje en abierto y en línea sean exitosas deben: a) responder a las motivaciones extrínsecas de los participantes; b) incluir contenidos que fomenten el estímulo intelectual, el compromiso y por ende los resultados de aprendizaje; y c) diseñar tareas y estrategias de evaluación con valoraciones y calificaciones humanas, dosificando la mediación tecnológica. Estas conclusiones son vitales para el diseño de cursos en línea dirigidos a docentes, que exigen varias consideraciones. Primero, disponer de información previa bajo sondeo de necesidades de los perfiles destinatarios. Segundo, disponer de guías claras explicando objetivos y competencias a alcanzar con la formación. Tercero, definir no solo modelos teóricos sino también razones y ejemplificaciones sencillas que permitan comprender por qué enseñar o utilizar una tecnología, entendiéndola más allá de herramienta como "reflexión sistemática y procesual sobre el saber técnico" (García-Aretio, 2020, p. 16). Y cuarto, buscar nuevos formatos para compartir buenas prácticas, como videos con anotaciones y etiquetado social en redes profesionales (Ruiz-Rey et al., 2021).

\section{Formatos masivos para el aprendizaje de emergencia}

Entre los diversos formatos audiovisuales de aprendizaje ágil y en abierto en red, los cursos de formación abiertos basados en nano-experiencias de aprendizaje o NanoMOOC o NOOC (combinación del inglés Nano Learning Experience y Open Online Course), de entre 1 y 20 horas de carga lectiva (Inzolia, 2018), materializan 
otra tendencia en expansión para la formación permanente o corporativa: el microlearning (Malamed, 2005). Esta estrategia, propia de la sociedad líquida (Bauman, 2013) y de las cambiantes exigencias del mercado laboral, surge para dar información just in time y respuesta ágil a demandas formativas específicas con píldoras de aprendizaje en línea de carácter breve (micro-contenidos) y, en su caso, actividades con objetivos de aprendizaje muy delimitados. Su auge está llevando a plantear incluso sistemas de micro-credenciales como oportunidades de aprendizaje flexible de calidad en las universidades del futuro (DEVA, 2021).

Junto a estos y otros modelos de carácter asíncrono, como los cursos virtuales tipo SPOC (del inglés Self-Paced Open Online Course), dirigidos a determinados colectivos, ganan fuerza micro-actividades formativas en directo que usan salas o aulas virtuales similares a las que el profesorado viene empleando en docencia bimodal o virtualizada ante la pandemia, con la diferencia de que en éstas la totalidad de la formación acontece a través de estas salas (Sánchez, 2020). Nos referimos a los webinars o webinarios, término que hoy alude a los eventos formativos por webconferencia, dirigidos y limitados a segmentos concretos de audiencia $\mathrm{u}$ ofertados a cualquier perfil, de forma gratuita o previo pago según los casos (Sánchez, 2020). Aunque existen precedentes de éxito, ha sido con la pandemia y las nuevas necesidades formativas fruto de la digitalización táctica de las actividades cuando diversas entidades han visto en estos formatos de aprendizaje abierto y en línea una solución rápida y eficaz para esta situación extrema (Schildkamp et al., 2020).

\section{Los webinars como microaprendizaje síncrono en línea}

Los webinars son una "estrategia formativa on line con alto potencial para ser utilizada por comunidades de expertos o especialistas de un mismo campo de interés profesional" (Area et al., 2014, p. 23), que además no requiere de grandes inversiones. Desde hace años viene aplicándose como fórmula para el aprendizaje a lo largo de la vida por universidades y organizaciones, ya sea para ofertar formación externa o capacitar a su personal. $Y$ en distintos niveles educativos, los seminarios con expertos son a menudo excelente complemento a lo aprendido en el aula, o fórmula para fomentar el aprendizaje activo y las competencias básicas de los estudiantes cuando éstos realizan sus propias presentaciones en línea, con resultados muy positivos en cuanto a valoración y rendimiento académico (Area et al., 2014; Peinado et al., 2019; Gegenfurtner et al., 2020).

Si bien no siempre se aprovecha la comunicación bidireccional y el potencial interactivo de la webconferencia frente a sistemas de streaming tradicionales y más orientados a la mera retransmisión, desde la perspectiva educativa los webinars suelen caracterizarse por varios rasgos (Sánchez, 2020). Así, son formatos multimedia y telepresenciales, que humanizan la formación en línea mediante imagen y audio en tiempo real, con herramientas que permiten, aparte de exponer compartiendo presentaciones o determinado contenido desde la pantalla, interaccionar y trabajar 
de forma colaborativa en tiempo real. Integran además opciones que facilitan tanto la gestión del directo (personalización de accesos y permisos/asignación de roles) como la posterior generación de contenidos (grabación de sesiones).

\section{Fórmulas para la capacitación docente ante la sobrevenida enseñanza en línea por la Covid-19 en el contexto de la Universidad Internacional de Andalucía}

Durante 2020 y 2021 numerosas entidades vienen organizando bajo la denominación de webinars actividades diversas, algunas previstas inicialmente como presenciales y otras nacidas como respuesta a demandas coyunturales fruto de la Covid-19, a menudo abiertas y gratuitas como estrategia de visibilización de marcas o captación de usuarios. Basta con hacer una rápida comprobación a través de herramientas como Google Trends y los propios buscadores de redes sociales para comprobar los numerosos resultados que arroja el término webinar en este periodo, asociado también a eventos divulgativos, científicos e incluso profesionales más allá de la academia.

Tampoco ha quedado al margen la capacitación del profesorado universitario, que ha encontrado en estas fórmulas una oportunidad para dar respuesta ágil, síncrona y ciertamente masiva a las demandas formativas en cuanto a e-competencias e innovación educativa fruto de la virtualización sobrevenida de la docencia ante la pandemia. Es momento de evaluar la eficacia y eficiencia de dichas acciones: no solo porque la pandemia continúa sino porque se espera que, tras ésta, sigan presentes estas tecnologías en la enseñanza como lo estaban antes en las universidades "más digitales".

Este artículo se centra en los llamados \#webinarsUNIA, seminarios por webconferencia sobre e-learning, innovación educativa y competencias digitales, de la Universidad Internacional de Andalucía. Una iniciativa pionera e innovadora de capacitación docente, iniciada en 2013-14 dentro de sus planes anuales de formación de profesorado, pero abierta a la participación de cualquier persona interesada.

Se ha venido desarrollando con éxito en cuanto a inscripciones y satisfacción de los participantes, llegando incluso a recibir reconocimientos externos vinculados a responsabilidad social ${ }^{1}$. Ha contribuido además a la generación de conocimiento abierto más allá del directo, puesto que grabaciones y presentaciones de los webinars se han ido publicando bajo Creative Commons en el repositorio institucional de la universidad, donde a fecha de junio de 2021 ya hay disponibles unos 6o REA. Y ha supuesto además (y aquí otra de sus innovaciones, como se comenta más adelante) una oportunidad para testear nuevos sistemas de microcredenciales digitales y conformar itinerarios formativos en red. De carácter masivo e internacional, los \#webinarsUNIA son seguidos por participantes de diversos territorios, más allá de España, especialmente latinoamericanos, sumando la edición más reciente, curso 
2020-21, cerca de 16.000 inscripciones (Universidad Internacional de Andalucía, 2021).

Partimos de estudios previos a la Covid-19, que identificaban ya las competencias digitales de los docentes, como "DigCompEdu" (Redecker, 2017); de informes de la Comisión TIC en la CRUE (CRUE, 2021) y de estudios específicos en la enseñanza universitaria sobre la competencia digital de los docentes en todas las áreas (CaberoAlmenara, et al., 2021).

Nos centramos en la metodología seguida para el diseño y evaluación de estos webinars de 2020-21 y en los resultados de una iniciativa que ha sido además germen para nuevos proyectos innovadores de aprendizaje abierto y en línea de la UNIA. Son por tanto objetivos de este estudio:

a. Evaluar el diseño y contenidos del programa de \#webinarsUNIA desde la perspectiva de los usuarios finales.

b. Disponer de contenidos e ideas para diseñar nuevos programas formativos en abierto en formatos MOOC y SPOC desde la evaluación de estos webinars.

c. Aportar, a partir de las fortalezas y aspectos de mejora detectados en esta experiencia, claves para el diseño de programas de capacitación docente en abierto de utilidad para otros profesionales y entidades.

\section{MÉTODO}

\section{Selección de temáticas y clasificación de los webinars}

La selección de temáticas y el enfoque de los \#webinarsUNIA de 2020-21 se realizó tomando como base los resultados de la encuesta anual de necesidades y expectativas formativas que se suministra al profesorado de la Universidad y las competencias básicas de interés para ese momento crucial como fueron, por números según el DigCompEdu ya mencionado: 2. Recursos Digitales 3. Pedagogía Digital 4. Evaluación y Retroalimentación, 6. Facilitar la Competencia Digital (como puede observar en la Tabla 1); se consideraron también especialmente las demandas que plantearon los responsables de programas cuando las personas de innovación y enseñanza virtual les contactaron para darle el apoyo que necesitaban en su tránsito repentino de lo presencial a lo virtual (marzo 2020).

Fueron 20 webinars los diseñados según lo anterior, impartidos entre junio de 2020 y febrero de 2021. Los primeros se centraron en atender cuestiones "urgentes", como el modo de evaluar en línea dada la cercanía de los exámenes finales, claves para planificar y adaptar programas formativos a e-learning, o posibilidades educativas de la webconferencia y consejos para organizar e impartir ante la cámara sesiones síncronas en línea. El ciclo concluyó con algunos seminarios orientados a explorar formas y tendencias sobre aprendizaje abierto en línea. Se establecieron, así, cinco grupos o categorías para clasificar los seminarios de 2020-21 (ver tabla inferior): 


\section{Tabla 1}

Listado de categorías temáticas y webinars de 2020-21 asociados

\section{Categoria}

\section{$n^{0}$ y títulos de los webinars}

1. "Proctoring para la evaluación en tiempos de Covid-19"

2. "Evaluación formativa en escenarios digitales"

3. "Cómo plantear la docencia como innovación"

Diseño de programas en línea, modelos y metodologías (12 webinars) sobre el modelo de la UNIA" posibilidades y organización de actividades"
4. "Diseñar/ adaptar programas formativos a e-learning: claves

5. "La Webconferencia para el aprendizaje síncrono en red:

6. "Flipped classroom en docencia universitaria"

7. "Gamificación para entornos virtuales"

8. "Vídeos y podcasts para humanizar la experiencia de estudiantes en línea"

9. "Presentaciones y contenidos gamificados para activar el aprendizaje"

Diseño de programas en línea, modelos y metodologías (12 webinars)

10. "Anotaciones colaborativas sobre vídeos y audios"

11. "Rúbricas digitales: creación y posibilidades como herramienta de evaluación"

12. "Planificar y acompañar con éxito en tutorías en red"

13. "Design thinking en educación"

Herramientas
de innovación y emprendimiento aplicadas a educación ( 2 webinars)

14. "Modelo Canvas para aprendizaje basado en proyectos y diseño de proyectos de innovación"

Tecnologías innovadoras 15. "Realidad Aumentada, modelado en 3D e impresión en 3D al servicio de la para construir conocimiento"

educación (2 webinars) 16. "Robótica educativa"

Creación de contenidos y otras competencias

17. "Estrategias para el diseño de materiales y recursos de aprendizaje accesibles"

digitales (2 webinars)

18. "Trucos para automatizar Moodle como docente"

19. "Repositorios universitarios al servicio de la enseñanzaaprendizaje"

línea (2 webinars).
20. "Claves para el diseño e impartición de MOOC y derivados"

Fuente: elaboración propia. 


\section{Estructura y desarrollo de cada actividad}

El esquema seguido para cada seminario fue el mismo. Una sesión en directo por webconferencia, empleando una sala virtual que daba cabida hasta a 1.000 participantes simultáneos, en la que, tras presentar la actividad, un ponente realizaba una exposición del contenido de 1-1,5 horas aproximadamente y tras ello se dejaba turno a preguntas, moderadas por personal de Innovación de la Universidad. Más allá del directo, las sesiones se grabaron y se pusieron a disposición de los participantes a través de un espacio en el Campus Virtual de la Universidad, donde tenían además un test cuya superación les posibilitaba descargar una micro-credencial de participación. En paralelo, estas grabaciones y las presentaciones empleadas se fueron subiendo a canales en red de la Universidad e incorporándose al repositorio digital institucional, quedando disponibles de forma abierta para su uso y reutilización por personas más allá de los participantes. Todo el sistema de producción, dinamización y acreditación, así como su agilidad y rápida adaptación a las necesidades de una comunidad de usuarios en aumento, hacen singular e innovadora la iniciativa. Otras universidades vienen mostrando su interés antes y, sobre todo, con la pandemia, sumando a su profesorado como participante y/o solicitando asesoramiento para transferir el modelo (especialmente el sistema de mini-acreditación automática y la replicación de contenidos).

\section{Sistema de seguimiento y evaluación de la iniciativa}

Como para el resto de líneas de formación de profesorado de la Universidad, durante su planificación se definió un sistema de seguimiento y evaluación individual (por actividad) y global (del conjunto de los \#webinarsUNIA) con distintos indicadores. Algunos eran relativos a la difusión e impacto de la iniciativa como aprendizaje abierto en línea. En lo estrictamente vinculado a la formación se tuvo en cuenta número de participantes, tasa de abandono o rendimiento académico, nivel de satisfacción de participantes y cumplimiento del desarrollo previsto en cada uno de los seminarios, analizando luego los datos en conjunto.

Para recoger estos datos se emplearon fundamentalmente dos instrumentos. Por un lado, a través de las inscripciones (formulario y base de datos en línea), de las que se obtuvieron variables como el perfil profesional o la procedencia geográfica de los participantes. Y por otro, mediante el suministro de encuestas en línea tras cada webinar. Las encuestas contenían unas 15 preguntas, la mayoría cerradas, dicotómicas o de Escala Likert de valoración, orientadas a la evaluación de cada actividad. Organizadas por bloques, cubrían las siguientes dimensiones:

- Organización y coordinación del programa: percepción acerca de la antelación de la convocatoria e indicaciones previas; de la duración y horario del webinar; o de la información proporcionada. 
- Actuación docente: valoración de la calidad de la exposición, dominio de la materia, atención a dudas/preguntas y claridad en expresar ideas.

- Aspectos técnicos y experiencia con la sala para el directo o campus virtual de la Universidad para el "post-webinar" (percepción, dificultades...).

- Generales: expectativas iniciales y motivos para participar en el webinar (incluyendo en qué grado la acreditación supone una razón para hacerlo); cumplimiento de expectativas (sí/no y por qué); percepción del webinar como herramienta de aprendizaje; o valoración de aspectos más positivos y sugerencias de mejora (preguntas abiertas).

Para facilitar la comparativa con ediciones anteriores, en 2020-21 se mantuvo idéntico el modelo de encuestas. La respuesta a éstas era de carácter voluntario. Algunos resultados se analizaron de forma cualitativa, al tiempo que se realizó un análisis estadístico, mediante el software SPSS versión 21.0, de ciertas variables conforme se detalla a continuación.

\section{Muestra}

En total hubo 10.933 inscritos (con una media de 547 por webinar, frente al promedio de 144 de 2019-20). De estos, más de la mitad $(51,88 \%, 5.671)$ logró concluir con éxito los seminarios y obtener la acreditación -recordemos, voluntaria y autodescargable tras superar un test de evaluación desde el Campus Virtual de la Universidad-..En cuanto a perfiles, la mayoría deinscritos eran docentesuniversitarios de otras universidades a la organizadora (alrededor del 50\%). Había también docentes de otros niveles educativos como Secundaria y FP (13\%); personal no docente de instituciones educativas (7-8\% aproximadamente); formadores/consultores de formación empresarial (en torno a un 5\%); y otros colectivos (porcentaje restante): estudiantes de diferentes niveles universitarios (grado, posgrado o doctorado) vinculado a educación u otras áreas; técnicos de los departamentos de formación y personal de administración -PAS- de distintas instituciones; investigadores y docentes de distintas universidades. Hubo participación desde 35 países diferentes; si bien, el grueso de la muestra se inscribió desde España (algo más de un 70\%), buena parte procedía del ámbito Iberoamericano (cerca de un 23\%), de más de una veintena de países distintos. Hallamos también, en menor medida, inscripciones de otros países europeos (sobre el 2\%), de EE.UU. y de países asiáticos como Corea del Sur y Japón.

Del total de participantes, cumplimentaron los cuestionarios de valoración algo más de 1900 personas (ver tabla inferior). Si bien podría entenderse como una muestra reducida, podemos considerarla válida interpretándose respecto al número de personas que superó cada webinar en lugar de a la cantidad de inscritas. 


\section{Tabla 2}

Tasa de respuesta a encuestas de valoración de webinars

\begin{tabular}{ccc} 
& $n^{0}$ del webinar & Respuestas a encuesta de valoración final \\
\cline { 3 - 3 } (ver títulos completos en Tabla 1) & No & $\begin{array}{c}\text { \% respecto a quienes } \\
\text { superaron el webinar }\end{array}$
\end{tabular}

\begin{tabular}{|c|c|c|}
\hline 1. "Proctoring...". & 101 & $69,1 \%$ \\
\hline 2. “Evaluación....”. & 191 & $65,2 \%$ \\
\hline 3. "Cómo plantear...". & 133 & $48,5 \%$ \\
\hline 4. “Diseñar/ adaptar...”. & 108 & $39,9 \%$ \\
\hline 5. "La Webconferencia...". & 105 & $35 \%$ \\
\hline 6. “Flipped classroom...”. & 153 & $44,8 \%$ \\
\hline 7. “Gamificación...”. & 124 & $35,4 \%$ \\
\hline 8. "Vídeos y podcasts...". & 121 & $36,5 \%$ \\
\hline 9. “Presentaciones...". & 81 & $24,1 \%$ \\
\hline 10. "Design thinking...". & 66 & $24,1 \%$ \\
\hline 11. "Anotaciones...". & 47 & $21,6 \%$ \\
\hline 12. "Rúbricas digitales...”. & 67 & $24,2 \%$ \\
\hline 13. "Planificar y acompañar...”. & 52 & $16,1 \%$ \\
\hline 14. "Modelo Canvas..." & 65 & $24,5 \%$ \\
\hline 15. "Realidad Aumentada...". & 42 & $23,2 \%$ \\
\hline 16. "Robótica educativa". & 61 & $27,7 \%$ \\
\hline 17. “Estrategias ...”. & 80 & $27,1 \%$ \\
\hline 18. “Trucos...". & 148 & $41,3 \%$ \\
\hline 19. "Repositorios...". & 80 & $26,1 \%$ \\
\hline 20."Claves...". & 83 & $26,3 \%$ \\
\hline Total de respuestas a encuestas & 1908 & $34,0 \%$ \\
\hline
\end{tabular}

Fuente: elaboración propia a partir de encuestas de valoración.

\section{Análisis cuantitativo de resultados}

Se puso el foco en los cuatro ítems relativos a la actuación docente (todos ellos con Escala Likert de o a 5), estudiando su fiabilidad y realizando un análisis de factores confirmatorio y comparaciones de las medias en función de la categoría o tipo de webinar. También se estudió mediante Likert un quinto ítem que medía la importancia de la acreditación para los participantes en los diferentes seminarios, observando asimismo si existían diferencias significativas en las medias obtenidas en las diferentes categorías.

Para el análisis de fiabilidad (esto es, comprobar hasta qué punto los ítems analizados estaban midiendo lo mismo) se propuso el coeficiente alfa de Cronbach 
con el resultado de un valor de 0,789 , lo que confirma que el estudio es consistente. Respecto al análisis de factores, se vio necesario analizar la validez del constructo considerando que el mismo "intenta determinar en qué medida un instrumento mide un evento en términos de la manera como éste se conceptualiza, y en relación con la teoría que sustenta la investigación" (Hurtado, 2012). La evidencia de la validez del constructo se obtuvo mediante el análisis de factores. Tal método agrupa los ítems en función de sus correlaciones, nos indica cuántas dimensiones integran a una variable y qué ítems conforman a cada dimensión. Los ítems que conforman una dimensión tienen altas correlaciones entre sí, agrupándolos estos ítems como un factor. Cuando los reactivos no pertenecen a una dimensión quiere decir que están aislados y no miden lo mismo que los demás ítems; por lo tanto, deben eliminarse.

Se realizaron los siguientes análisis: determinación de la matriz de correlaciones; índice KMO (Kaiser-Meter-Olkin) o medida de adecuación de la muestra; y test de esfericidad de Barlett. El índice KMO se interpreta de manera semejante a los coeficientes de confiabilidad, vale decir, con un rango de o a 1 y tomando como adecuado un valor igual o superior a 0,70 , el cual sugiere una interacción satisfactoria entre los ítems (Hair et al., 1999), obteniendo en nuestro estudio un valor de o,818. En el caso de que el índice KMO sea satisfactorio podemos utilizar el modelo de extracción de factores de componentes principales que consiste en llevar a cabo la combinación lineal de todas las variables de modo que el primer componente principal sea una combinación que explique la mayor proporción de varianza de la muestra, la segunda mayor y así sucesivamente. El objetivo es encontrar un reducido número de componentes que expliquen el máximo de varianza total de las variables originales.

Por último, el análisis de varianza (ANOVA) de un factor nos sirvió para comparar varios grupos en una variable cuantitativa. Se aplica para contrastar la igualdad de medias de tres o más poblaciones independientes y con distribución normal. Se considera que las diferencias de medias son significativas entre las medias de varios grupos si el nivel de significación es menor que 0,05.

\section{RESULTADOS}

\section{Valoración de los docentes y acreditación como motivación}

Como se anticipó, se efectuó un análisis estadístico cuantitativo, en el que se incluían 5 ítems (4 de los cuales valoraban la calidad de los ponentes de los webinar y 1 referido al interés por obtener la acreditación), usando el parámetro alfa de Cronbach; el análisis de factores confirmatorio; y un estudio comparativo de medias basado en el test de ANOVA en función de las categorías de los webinars. 


\section{Tabla 3}

Varianza total explicada. Método de extracción: Análisis de Componentes principales

\begin{tabular}{|c|c|c|c|c|c|c|}
\hline \multirow[t]{2}{*}{ Componente } & \multicolumn{3}{|c|}{ Autovalores iniciales } & \multicolumn{3}{|c|}{$\begin{array}{l}\text { Sumas de las saturaciones al } \\
\text { cuadrado de la extracción }\end{array}$} \\
\hline & Total & $\begin{array}{c}\% \text { de la } \\
\text { varianza }\end{array}$ & $\begin{array}{c}\% \\
\text { acumulado }\end{array}$ & Total & $\begin{array}{c}\% \text { de la } \\
\text { varianza }\end{array}$ & $\begin{array}{c}\% \\
\text { acumulado }\end{array}$ \\
\hline 1 & 3,581 & 89,524 & 89,524 & 3,581 & 89,524 & 89,524 \\
\hline 2 & ,208 & 5,201 & 94,725 & & & \\
\hline 3 & ,173 & 4,316 & 99,040 & & & \\
\hline 4 & ,038 &, 960 & 100,000 & & & \\
\hline
\end{tabular}

Fuente: elaboración propia

La varianza total explicada de la tabla anterior recoge un único factor en el que se integran los ítems que se refieren a las valoraciones de los ponentes.

En cuanto a las valoraciones generales de los ponentes, los promedios obtenidos sobre Escala Likert de o a 5 son elevados, situándose sobre 4,5 en todos los aspectos (claridad de la expresión de ideas; atención a dudas; dominio de la materia; y calidad de la exposición), destacando ligeramente el dominio de la materia.

\section{Figura 1}

Valoración media de los conferenciantes

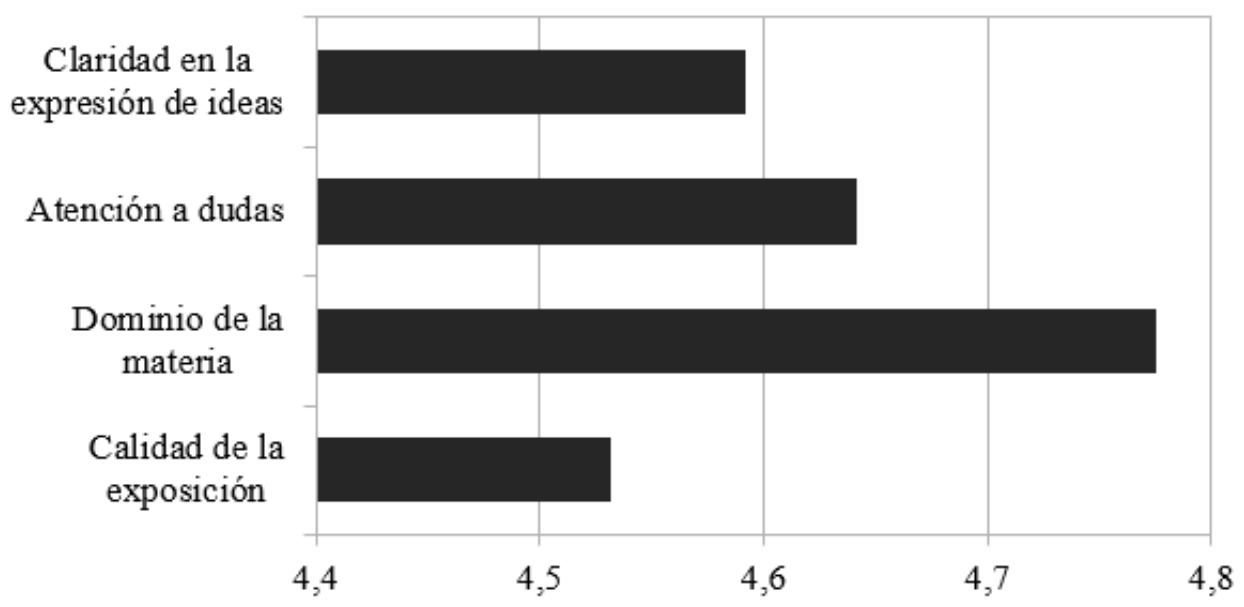

Fuente: elaboración propia. 
Por último, se analizaron cuantitativamente las posibles diferencias de medias en las valoraciones de ponentes y de la acreditación en función de las cinco categorías de webinars fijadas. Para ello se utilizó el test ANOVA junto con el HSD de Tukey, obteniéndose que las diferencias de medias respecto a las categorías de webinars no son significativas en ambos casos por obtener valores de significación mayores que 0,05. Más concretamente, en la tabla de medias de la valoración de la importancia de la acreditación en función de las cinco categorías de webinars establecidas se observa que las diferencias entre ellas no son significativas, lo que confirma el análisis de ANOVA y el HSD de Tukey mencionado.

\section{Tabla 4}

Medias de la valoración de la importancia de la acreditación según la categoría del webinar

\begin{tabular}{lc} 
Categoría del webinar & $\begin{array}{c}\text { Media de la valoración } \\
\text { de la importancia de la } \\
\text { acreditación }\end{array}$ \\
\hline Diseño de programas en línea, modelos y metodologías & 3,03 \\
\hline $\begin{array}{l}\text { Herramientas de innovación y emprendimiento aplicadas a } \\
\text { educación }\end{array}$ & 2,99 \\
\hline Tecnologías innovadoras al servicio de la educación & 3,22 \\
\hline Creación de contenidos y otras competencias digitales & 3,08 \\
\hline Aprendizaje abierto en línea & 3,31 \\
\hline
\end{tabular}

Fuente: elaboración propia.

Por otra parte, al ser preguntados sobre las acreditaciones por seguir los webinars como posible motivación, los valores obtenidos en las medias no son muy altos en la Escala Likert, lo que implica que los encuestados no dan mucha importancia a esta posibilidad.

\section{Razones para inscribirse y expectativas iniciales}

Entre las motivaciones que han llevado a los usuarios a inscribirse en los distintos webinars, junto a las asociadas al propio formato y sus ventajas destacan, sobre todo, en las encuestas, razones formativas como las siguientes:

- Necesidad de adaptación a nuevos escenarios docentes.

- Deseo de mejorar el nivel de las clases.

- Obtener mayor conocimiento sobre la enseñanza a distancia.

- Mejorar competencias digitales y docentes.

- Conocer mejor la temática concreta del webinar. 
- Por necesidades docentes derivadas de la situación de pandemia de la Covid-19.

- Actualizar conocimientos y mejorar la práctica docente.

- Acceso al conocimiento de metodologías activas.

La posibilidad de mejorar el currículum vitae o de obtener una acreditación aparecen también reseñadas, en menor medida, junto al carácter gratuito de los webinars y la experiencia/ prestigio de los ponentes.

\section{Satisfacción global y percepción sobre adecuación del formato}

En la siguiente tabla se recogen las apreciaciones del nivel de satisfacción de los usuarios con los 20 webinars impartidos, observándose unos porcentajes de satisfacción muy representativos. En la práctica totalidad el cumplimiento de expectativas se sitúa por encima del $90 \%$ e incluso del $95 \%$, hecho que avala los resultados a otra de las cuestiones, referida a la percepción sobre estos seminarios y la forma en que se han planteado (incluyendo organización y atención a personas inscritas, duración y horarios de las sesiones en directo) para el aprendizaje sobre enseñanza virtual e innovación.

\section{Tabla 5}

Cumplimiento de expectativas de los webinars de 2020-21

\begin{tabular}{|c|c|c|}
\hline $\begin{array}{l}\text { Denominación de webinar } \\
\text { (ver títulos completos en Tabla 1) }\end{array}$ & $\begin{array}{l}\text { Cumplimiento de } \\
\text { expectativas } \\
\text { (\% partic.) }\end{array}$ & $\begin{array}{c}\text { Percepción de } \\
\text { adecuación del } \\
\text { formato ( } \% \text { partic.) }\end{array}$ \\
\hline 21. "Proctoring...”. & $92,0 \%$ & $98,0 \%$ \\
\hline 22. "Evaluación....”. & $95,8 \%$ & $98,4 \%$ \\
\hline 23. “Cómo plantear...". & $94,7 \%$ & $94,0 \%$ \\
\hline 24. “Diseñar/ adaptar...”. & $96,0 \%$ & $99,0 \%$ \\
\hline 25. “La Webconferencia...”. & $97,1 \%$ & $99,0 \%$ \\
\hline 26. “Flipped classroom...”. & $96.1 \%$ & $98,7 \%$ \\
\hline 27. “Gamificación...”. & $89,5 \%$ & $100 \%$ \\
\hline 28. "Vídeos y podcasts...”. & $95,0 \%$ & $100 \%$ \\
\hline 29. “Presentaciones...”. & $87,5 \%$ & $92,8 \%$ \\
\hline 30. "Design thinking...”. & $95,5 \%$ & $97,1 \%$ \\
\hline 31. “Anotaciones...”. & $95,7 \%$ & $100 \%$ \\
\hline 32. "Rúbricas digitales...”. & $97 \% \%$ & $98,6 \%$ \\
\hline 33. "Planificar y acompañar...”. & $100 \%$ & $100 \%$ \\
\hline
\end{tabular}




\begin{tabular}{lll}
$\begin{array}{c}\text { Denominación de webinar } \\
\text { (ver títulos completos en Tabla 1) }\end{array}$ & $\begin{array}{c}\text { Cumplimiento de } \\
\text { expectativas } \\
\text { (\% partic.) }\end{array}$ & $\begin{array}{c}\text { Percepción de } \\
\text { adecuación del } \\
\text { formato ( \% partic.) }\end{array}$ \\
\hline 34. "Modelo Canvas...” & $95,4 \%$ & $100 \%$ \\
\hline 35. "Realidad...”. & $95,2 \%$ & $100 \%$ \\
\hline 36. "Robótica educativa". & $95,1 \%$ & $100 \%$ \\
\hline 37. "Estrategias...”. & $98,8 \%$ & $100 \%$ \\
\hline 38. “Trucos...". & $91,9 \%$ & $99,3 \%$ \\
\hline 39. "Repositorios...”. & $95,0 \%$ & $98,8 \%$ \\
\hline 40. “Claves...”. & $99,0 \%$ & $98,0 \%$ \\
\hline
\end{tabular}

Fuente: elaboración propia a partir de resultados de encuestas.

El análisis cualitativo muestra, además, que los participantes percibieron como aspectos más positivos la buena organización, la calidad de los materiales presentados, la calidad de los ponentes y el interés de los temas. Por otro lado, entre potenciales mejoras se propone seguir incidiendo en metodologías activas y emergentes, además de revisar algunos aspectos relacionados con la acreditación y la carga de presentaciones de los ponentes.

\section{Valoración de aspectos técnicos y organizativos}

Las valoraciones cualitativas de aspectos técnicos y organizativos apoyan también los resultados anteriores. Así, prácticamente la totalidad de asistentes consideró que se les convocó con tiempo suficiente y valoró muy positivamente las indicaciones previas de conexión vía e-mail. Una gran mayoría de usuarios conectó en directo, salvo algunos que no pudieron hacerlo por incompatibilidad horaria, por cuestiones laborales o por problemas de conectividad. Se valoró muy positivamente la duración del directo de los seminarios y la elección de horario (salvo determinados asistentes con huso horario diferente), y no se apreciaron problemas técnicos reseñables en el acceso a los webinars. En las respuestas abiertas muchos reseñan además la atención continua y personalizada por parte de la organización como otro de los motivos de su satisfacción con la experiencia.

\section{CONCLUSIONES}

En medio de un escenario pandémico, de sobreabundancia de formación gratuita en línea y de uso y "abuso" del término webinar por parte de diversos actores, la calidad de este tipo de formación es más importante que nunca para diferenciarse, sumar inscritos y fidelizarlos. Y especialmente, porque desde un buen modelo de 
webinar, los docentes podrán tomar ejemplos para una buena práctica en su uso para sus contextos, y obtengan mayor satisfacción los estudiantes de las generadas durante la pandemia en algunos casos (Roig-Vila et al., 2021) y que antes de la pandemia parece que tenía más reconocimiento (Peinado et al., 2019). Nos queda sin duda tomar más perspectiva e investigar en este formato en el futuro para despejar estas variables influyentes y la gran cantidad de fórmulas y tecnologías que entran dentro de lo que se considera webinar (Agaltsova et al., 2020).

Lo que comenzó siendo un programa de formación en abierto e innovador en aquellas fechas (curso 2013-14) en nuestro contexto, y en cierta forma experimental, se ha convertido en el curso 2020-21 en una estrategia de formación de profesorado de "urgencias" propia del "estado de excepción" que vivimos con la pandemia que ha dado buenos frutos en cuanto a inscripciones y valoración de los participantes.

La experiencia de los \#webinarsUNIA desde sus inicios hasta hoy, lleva a afirmar que se ha generado una comunidad de usuarios interesados en aprender sobre enseñanza virtual, innovación y competencias digitales. Esta comunidad originada desde las primeras ediciones de los \#webinarsUNIA se ha visto incrementada por numerosos docentes universitarios debido a la necesidad de virtualización de la docencia fruto de la Covid-19. Todos han encontrado en este programa formativo una oportunidad de aprendizaje ágil y flexible, con el añadido de que son abiertos, gratuitos y permiten la posibilidad de obtener una credencial de participación rápida y ágil.

Como apuntaban algunos participantes en las encuestas de valoración, iniciativas como estos webinars "permiten al docente renovarse y buscar nuevos métodos de docencia más atractivos para el estudiante" y son en este sentido "una grandísima propuesta para poder avanzar en la docencia”. El diseño de productos formativos breves, directos, prácticos y dinámicos se vuelve esencial en un tiempo en que los docentes y otros profesionales vinculados a la educación tienen problemas y cuestiones concretas que resolver para su labor de enseñanza en línea.

Los resultados de la evaluación realizada vienen a confirmar que la parte organizativa, la gestión y el acompañamiento a docentes y participantes de los webinars antes, durante y después del directo, resulta esencial para el éxito de estas iniciativas. El otro gran pilar es la calidad de la actuación docente. Es decir, por un lado, realizar una buena "curación" de contenidos, con casos prácticos y recursos complementarios para profundizar, y presentarlos en un formato atractivo y adaptado a la pantalla. Y por otro, tener capacidad para transmitir ante la cámara y responder a las dudas de los participantes durante la sesión. Se trata, en síntesis, de "humanizar" experiencias de aprendizaje de carácter masivo como ésta, prestando atención a la diversidad, ofreciendo la posibilidad de consultar el material más allá del directo y generando, en fin, recursos educativos en abierto al servicio de la comunidad universitaria y la sociedad en general.

Todo ello, en el contexto de instituciones que, como la Universidad Internacional de Andalucía, viene apostando por la innovación en la formación al profesorado, el 
aprendizaje abierto y en línea, abre además puertas para poner en marcha nuevos proyectos que den respuesta a las demandas sociales. Así, sobre los webinars de 202021 mejor evaluados y añadiendo otros materiales elaborados por los mismos expertos, se diseñó, se creó e impartió a finales de 2020 una primera edición de un curso tipo SPOC que tenía como objetivo formar para la docencia innovadora de programas en línea. Su diseño está también avalado por estudios como los anteriormente citados (Deng et al., 2020), y toma como ejes las "motivaciones extrínsecas de los participantes" (las preguntas y dudas más acuciantes manifestadas para virtualizar los programas en plena pandemia) y "las tareas y estrategias de evaluación realizadas por humanos" (solicitamos un proyecto individual de aplicación práctica de los modelos aprendidos, orientados y evaluados por cuatro tutoras). Con cerca de 200 participantes y 3 meses de duración, sus resultados positivos en cuanto a valoración abren además la posibilidad de conformar nuevos itinerarios formativos sobre TICs, e-learning o innovación en otros formatos tipo MOOC.

La experiencia técnica y organizativa de los \#webinarsUNIA aquí narrada, sirvió también de modelo para otras actividades, más allá de formación de profesorado, lanzadas por la Universidad durante la pandemia, como los llamados \#DiálogosUNIA, ciclo de seminarios por videoconferencia en abierto para reflexionar a través del debate con expertos sobre las consecuencias de la Covid-19 en distintos ámbitos, entre ellos, la educación en tiempos de pandemia. Lo que comenzó siendo una innovación es hoy una estrategia institucionalizada, con la que más allá de dar respuesta a la pandemia y atender las necesidades formativas de un público diverso y disperso geográficamente, se contribuye a la visión de una universidad abierta, innovadora y social tan necesaria hoy.

\section{NOTAS}

1. En concreto, el proyecto fue ganador de la categoría RSC de los Premios del Club de Marketing de Málaga 2017.

\section{REFERENCIAS}

Agaltsova, D. V., Rodinova, N., Beisenovna, S. R., Kagosyan, A. S., y Ostroukhov, V. (2020). Webinar as an innovative technology of online education with the use of modern media resources. Revista Inclusiones, 7(11), 119-132. https:// revistainclusiones.org/index.php/inclu/ article/view/1149

Albelbisi, N., Yusop, F. D., y Salleh, U. K. M. (2018). Mapping the Factors Influencing Success of Massive Open Online Courses
(MOOC) in Higher Education. Eurasia Journal of Mathematics, Science and Technology Education, 14(7), 2995-3012. https://doi.org/10.29333/ejmste/91486

Area, M., Sannicolás, B., y Borrás, J. F. (2014). Webinar como estrategia de formación online: descripción y análisis de una experiencia. Revista Latinoamericana de Tecnología Educativa - RELATEC, 13(1), 11-23. https://relatec.unex.es/article/ view/1197 
Bauman, Z. (2013). Sobre la educación en un mundo líquido. Paidós.

Bournissen, J., Cecilia-Tumino, M., y Carrión, F. (2019). MOOC: Evaluación y medición de la calidad percibida. International Journal of Educational Research and Innovation (IJERI), 11, 1832. https://www.upo.es/revistas/index. php/IJERI/article/view/2899/2946

Cabero-Almenara, J., Guillén-Gámez, F. F., Ruiz-Palmero, J., y Palacios-Rodríguez, A. (2021). Digital competence of higher education professor according to DigCompEdu. Statistical research methods with ANOVA between fields of knowledge in different age ranges. Education and Information Technologies. https://doi.org/10.1007/s10639-02110476-5

CRUE (2021). Portal informes CRUE. https://www.crue.org/informes-yposicionamientos/

Deng, R., Benckendorff, P., y Gannaway, D. (2020). Linking learner factors, teaching context, and engagement patterns with MOOC learning outcomes. Journal of Computer Assisted Learning, 30, 723. https://doi.org/10.1111/jcal.12437

DEVA (2021). Las micro-credenciales: oportunidades de aprendizaje flexible de calidad en las universidades del futuro. Jornadas organizadas por la Dirección de Evaluación y Acreditación, Agencia Andaluza del Conocimiento, Consejería de Transformación Económica, Industria, Conocimiento y Universidades, Junta de Andalucía, 24 de marzo de 2021. http:// deva.aac.es/jornadas/webinars/

Gallego-Arrufat, M.-J., Gamiz-Sanchez, V. M., y Gutiérrez-Santiuste, E. (2015). Tendencias en la evaluación del aprendizaje en cursos en línea masivos y abiertos. Educación $X X 1,18(2)$. https:// doi.org/10.5944/educxx1.12935

García-Aretio, L. (2020). Los saberes y competencias docentes en educación a distancia y digital. Una reflexión para la
formación.RIED.RevistaIberoamericana de Educación a Distancia, 23(2), 9-30. https://doi.org/10.5944/ried.23.2.26540

García-Peñalvo, F. J., Fidalgo-Blanco, Á., y Sein-Echaluce, M. L. (2017). Los MOOC: un análisis desde la perspectiva de la innovación institucional universitaria. $L a$ Cuestión Universitaria, 9, 117-135.

Gegenfurtner, A., Zitt, A., y Ebner, C. (2020). Evaluating webinar-based training: a mixed methods study of trainee reactions toward digital web conferencing. International Journal of Training and Development, 24(1), 5-21. https://doi. org/10.1111/ijtd.12167

Hair, J. F., Anderson, R. E., Tatham, R. L., y Black, W. (1999). Análisis Multivariante. Prentice Hall.

Hurtado, J. (2012). Metodología de la investigación: guía para una comprensión holística de la ciencia (4a. ed.). Ciea-Sypal y Quirón.

Inzolia, Y. (Coord.) (2018). Guía práctica para el diseño y tutorización de MOOC. Miriadax.net. https://miriadax.net/

Lemos-de-Carvalho-Junior, G., CebriánRobles, D., Cebrián-de-la-Serna, M., y Raposo-Rivas, M. (2019). Comparative Study SPOC vs. MOOC for SocioTechnical Contents from Usability and User Satisfaction. Turkish Online Journal of Distance Education, 4-20. https://doi. org/10.17718/tojde.557726

López-Meneses, E., Vázquez-Cano, E., y Román, P. (2015). Análisis e implicaciones del impacto del movimiento MOOC en la comunidad científica: JCR y Scopus (2010-13). Comunicar, 44(22), 73-80. https://doi.org/10.3916/C44-2015-08

Malamed, C. (2015). Is Microlearning The Solution You Need?. The elearning coach, 11 de octubre de 2016. http:// theelearningcoach.com/elearning 2-o/ what-is-microlearning/

Peinado, P., Bustillo, R., y Gálvez, C. (2019). Webinar: Una herramienta actual global al servicio del deep-learning presencial. 
Journal of Management and Business Education, 2(3), 279-292. https://doi. org/10.35564/jmbe.2019.0018

Raposo-Rivas, M., Martínez-Figueira, E., y Sarmiento-Campos, J. A. (2015). A Study on the Pedagogical Components of Massive Online Courses. Comunicar, 22(44), 27-35. https://doi.org/10.3916/ C44-2015-03

Redecker, C. (2017). Marco europeo para la competencia digital de los educadores. DigCompEdu. Comisión Europea. https://acortar.link/wU3qRH

Roig-Vila, R., Urrea-Solano M., y MermaMolina, G. (2021). La comunicación en el aula universitaria en el contexto del Covid-19 a partir de la videoconferencia con Google Meet. RIED. Revista Iberoamericana de Educación a Distancia, 24(1), 197-220. https://doi. org/10.5944/ried.24.1.27519

Ruiz-Rey, F. J., Cebrián-Robles, V., y Cebrián-de-la-Serna, M. (2021). Redes profesionales en tiempo de Covid19: compartiendo buenas prácticas para el uso de TIC en el prácticum. Revista Practicum, 6(1), 7-25. https://doi.org/10.24310/ RevPracticumrep.v6i1.12283

Sánchez, M. (2020). Webconferencia en educación: potencial, posibles usos, herramientas y organización de actividades. Guías prácticas Cibermarikiya.com. $\quad \underline{\text { htps://bit. }}$ ly/3eusM89

Schildkamp, K., Wopereis, I., Jong, M. K.-D., Peet, A., y Hoetjes, I. (2020). Building blocks of instructor professional development for innovative ICT use during a pandemic. Journal of Professional Capital and Community, 39, 88. https:// doi.org/10.1108/JPCC-06-2020-0034

Universidad Internacional de Andalucía (2021). Plan de Formación y Apoyo al Profesorado de la UNIA 2020-21. https:// www.unia.es/innovacion/formacionprofesorado/programas-anteriores

Yepes-Baldó, M., Romeo, M., Martín, C., García, M. Á., Monzó, G., y Besolí, A. (2016). Quality indicators: developing "MOOCs" in the European Higher Education Area. Educational Media International, 53(3), 184-197. https:// doi.org/10.1080/09523987.2016.123699 $\underline{8}$

Zancanaro, A., y Domingues, M. J. (2017). Analysis of the scientific literature on Massive Open Online Courses (MOOC) / Análisis de la literatura científica sobre los cursos en línea abiertos y masivos (MOOC). RIED. Revista Iberoamericana de Educación a Distancia, 2o(1), 59-80. https://doi.org/10.5944/ried.20.1.15910

\section{PERFIL ACADÉMICO Y PROFESIONAL DE LOS AUTORES}

María Sánchez González. Doctora en Periodismo por la Universidad de Málaga, España. Profesora asociada en Periodismo de la Universidad de Málaga desde 2010 y técnico responsable de formación de profesorado y aprendizaje abierto en red en Área de Innovación de la UNIA desde 2007. Con amplia experiencia como gestora, docente e investigadora en e-learning, innovación, cultura digital, comunicación y participación en red y planificación estratégica. Acreditada como profesora titular por ANECA. www.cibermarikiya.com. https://orcid.org/ooooo003-3053-0646

E-mail: $\underline{\text { m.sanchez@unia.es }}$ 
María Lourdes Miró Amarante. Ingeniera Informática (1999) y Doctora por la Universidad de Sevilla (2013). Profesora Contratada Doctora de la U. Sevilla, desde 2003. Directora de Secretariado Innovación Docente y Digitalización de la Universidad Internacional de Andalucía desde 2017. Coordinadora del Máster Ingeniería Biomédica y Salud Digital de U. Sevilla desde 2019; miembro del Grupo Asesor de Apoyo Pedagógico de Tecnologías Educativas y Recursos Digitales de U. Sevilla, desde 2020. https://orcid.org/o0oo-0002-7162-9776

E-mail: l.miro@unia.es

Francisco José Ruiz Rey. Doctor en Ciencias de la Educación por la UNED, licenciado en Ciencias Exactas por la Universidad de Málaga, experto en el uso de Internet y sus aplicaciones por la UNED y profesor asociado de la universidad de Málaga en el área de Didáctica de la Matemática desde el año 2014. Con amplia experiencia en la implementación de las TIC en las aulas y el uso educativo de dispositivos móviles. https://orcid.org/00o0-0002-5064-6534

E-mail: fruizrey@uma.es

Manuel Cebrián de la Serna. Catedrático Universidad de Málaga. Vicerrector de Innovación Docente y Digitalización UNIA. Líneas: a) innovación educativa vs. tecnológica; b) Enseñanza universitaria, y c) Prácticas externas. Dirige el grupo investigación Globalización, Tecnología, Educación y Aprendizaje (Gtea, SEJ-462) y la Revista Prácticum de la Asociación para el Desarrollo del Prácticum y de las Prácticas Externas: Red de Prácticum (REPPE). Ha sido director de diferentes centros de formación e innovación educativa y proyectos I+D+i. https://orcid. org/0000-0002-0246-7398

E-mail: m.cebrian@unia.es

\section{DIRECCIÓN DE LOS AUTORES}

Universidad Internacional de Andalucía. Sede Tecnológica de Málaga.

Plaza de la Legión Española, 1.

29007. Málaga, España.

Fecha de recepción del artículo: 30/04/2021

Fecha de aceptación del artículo: 25/08/2021

Fecha de aprobación para maquetación: 14/09/2021 